\title{
Hetero-oligomeric glutamate dehydrogenase from Thermus thermophilus
}

\section{Correspondence \\ Makoto Nishiyama \\ umanis@mail.ecc.u-tokyo.ac.jp}

Received 18 June 2010

Revised 6 September 2010

Accepted 7 September 2010

\author{
Takeo Tomita, ${ }^{1}$ Takashi Miyazaki, ${ }^{1}$ Junichi Miyazaki, ${ }^{1}$ \\ Tomohisa Kuzuyama ${ }^{1}$ and Makoto Nishiyama ${ }^{1,2}$
}

\author{
${ }^{1}$ Biotechnology Research Center, Department of Biotechnology, The University of Tokyo, Tokyo \\ 113-8657, Japan \\ ${ }^{2}$ RIKEN SPring-8 Center, Hyogo 679-5148, Japan
}

\section{INTRODUCTION}

Glutamate dehydrogenase (GDH) catalyses the reversible oxidative deamination of glutamate to produce 2-oxoglutarate (2-OG) and ammonium (Fig. 1) (Smith et al., 1975), providing a major pathway for the interconversion of $\alpha$-amino acids and 2-oxo acids. GDH is classified into three groups by coenzyme specificity: $\mathrm{NAD}^{+}$-dependent GDH (EC 1.4.1.2) (Camardella et al., 2002; Veronese et al., 1974), $\operatorname{NAD}(\mathrm{P})^{+}$ -dependent GDH (EC 1.4.1.3) (Baggio \& Morrison, 1996; Wen \& Morrison, 1996) and $\mathrm{NADP}^{+}$-dependent GDH (EC 1.4.1.4) (Aalén et al., 1997; Kobayashi et al., 1995). NAD ${ }^{+}$ -dependent GDHs are further classified into three subgroups according to the molecular size of a single subunit: GDH50s, GDH115s and GDH180s (Miñambres et al., 2000). GDH50s are known to have a homo-hexameric structure (Rice et al., 1987), while GDH115s have a homo-tetrameric structure (Veronese et al., 1974), and GDH180s have several forms with

Abbreviations: GDH, glutamate dehydrogenase; HTK, hyper-thermostable kanamycin nucleotidyltransferase; 2-OG, 2-oxoglutarate.

Four supplementary figures are available with the online version of this paper. homo-hexameric, homo-tetrameric or homo-dimeric structures (Kawakami et al., 2007; Lu \& Abdelal, 2001; Miñambres et al., 2000).

GDH50s are further classified into two subtypes: one is mammalian GDH, having an approximately 50 aa insertion that forms an antenna responsible for the heterotropic control, and the other is GDH lacking the antenna sequence. Mammalian GDH is regulated in a complex manner (Hudson \& Daniel, 1993). ADP, $\mathrm{NAD}^{+}$and leucine activate mammalian GDH, while GTP and NADH act as allosteric inhibitors. Mutations conferring resistance to GTP inhibition cause hyperinsulinism/hyperammonaemia syndrome in humans by increasing the ratio of ATP to ADP through activation of the tricarboxylic acid cycle by providing 2-OG, which in turn results in the stimulation of insulin secretion through closure of ATP-sensitive potassium channels and depolarization of beta cells for calcium influx (Stanley et al., 1998). Microbial GDH50s that lack the antenna have also been shown to be regulated. Fungal GDH50s from Neurospora crassa and Aspergillus species are regulated in a homotropic manner by glutamate, although they use $\mathrm{NADP}^{+}$as the coenzyme (Noor \& Punekar, 2005; West 


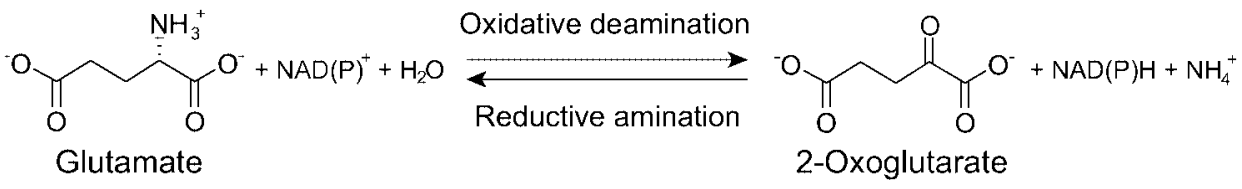

Fig. 1. Reactions catalysed by $\mathrm{GDH}$.

et al., 1967). The N. crassa GDH is also activated by nonsubstrate, di- or polycarboxylic acids (West et al., 1967), suggesting heterotropic regulation in this GDH. As for bacterial GDH, GDH from Clostridium symbiosum has been known to be regulated in a homotropic manner (Hamza \& Engel, 2008). Recently, heterotropic regulation has been reported for $\mathrm{NAD}^{+}$-dependent GDHs from bacteria such as Streptomyces clavuligerus (activation by AMP, aspartate and asparagine) (Miñambres et al., 2000), Janthinobacterium lividum (activation by aspartate and arginine) (Kawakami et al., 2007) and Pseudomonas aeruginosa (activation by arginine and inhibition by citrate) (Lu \& Abdelal, 2001). Thus, the presence of allosteric regulation suggests that GDH plays (an) important role(s) in metabolic control at the crossroads of carbon and nitrogen metabolism in various organisms.

Recent whole-genome sequencing has revealed that Thermus thermophilus HB8 (NCBI accession numbers NC_006461, NC_006462 and NC_006463) and HB27 (Henne et al., 2004) possess two putative $g d h$ genes on the genome, both of which encode GDH50s sharing approximately $46 \%$ identity in the amino acid sequence. Although GDH has been purified from T. thermophilus HB8 and characterized (Ruiz et al., 1998, 2003), it is not clear which isozyme was purified and characterized. Very recently, Bolivar and colleagues reported that recombinant $\mathrm{GDH}$ from $T$. thermophilus HB27, named GdhB in our present study, has GDH activity (Bolivar et al., 2008); however, the regulatory mechanism remains to be elucidated. Here, we report that the formation of a hetero-complex composed of inactive GdhA and active GdhB of T. thermophilus HB27 shows allosteric activation by hydrophobic amino acids.

\section{METHODS}

Strains, media and chemicals. Escherichia coli DH5 $\alpha$ (Sambrook et al., 1989) was used for DNA manipulation, and E. coli BL21Codon-Plus BL21(DE3)-RIL [F $\mathrm{F}^{-}, \operatorname{ompt}$, hsdS $\left(\mathrm{r}_{\mathrm{B}}^{-}, \mathrm{m}_{\mathrm{B}}^{-}\right), d c m^{+}, \mathrm{Tet}^{\mathrm{r}}$, gal, BL21(DE3), endA, Hhe, [argU, ileY, leuW, Cam ${ }^{\mathrm{r}}$ (Stratagene) was used as the host to express $g d h$ genes. The $2 \times$ yeast extract-tryptone (YT) (Sambrook et al., 1989) medium was generally used for cultivation of $E$. coli cells, whereas TM medium (Koyama et al., 1986) and minimal medium (MM) (Tanaka et al., 1981) were used for routine cultivation of T. thermophilus cells. Another medium, named E medium, containing $5 \mathrm{mM}$ Glu as both $\mathrm{C}$ and $\mathrm{N}$ sources instead of sucrose and $\mathrm{NH}_{4} \mathrm{Cl}$ in $\mathrm{MM}$ medium, was used to examine the in vivo function of the $g d h A$ and $g d h B$ genes. Antibiotics and IPTG were added to the medium when required. All chemicals were purchased from Sigma-Aldrich, Wako Pure Chemical or Kanto Chemicals. Enzymes for DNA manipulation were purchased from Takara Shuzo or Toyobo.

Expression of $\boldsymbol{g d h} \boldsymbol{A}$ and $\boldsymbol{g d h} \boldsymbol{h}$, and co-expression of $\boldsymbol{g d h} \boldsymbol{A}$ and $\boldsymbol{g d h} \boldsymbol{B}$ in $\boldsymbol{E}$. coli. To construct the plasmid for the expression of $g d h A$ or $g d h B$, each gene fragment was amplified from the genome of $T$. thermophilus HB27 by PCR with a pair of synthetic oligonucleotides, $g d h A(\mathrm{MCS} 1)-\mathrm{N} / g d h A(\mathrm{MCS} 1)-\mathrm{C}$ or $g d h B(\mathrm{MCS} 1)-\mathrm{N} / g d h B(\mathrm{MCS} 1)-\mathrm{C}$, respectively, listed in Table 1 . The amplified fragments were digested with BamHI/HindIII for $g d h A$ and EcoRI/SalI for $g d h B$ and cloned into pBlueScript II SK $(+)$. After the nucleotide sequence was verified, each DNA fragment was cloned into the multiple cloning site (MCS) 1 of pETDuet-1 (Novagen). The resulting plasmids, pETD1-gdhA(his) and pETD1-gdhB(his), were designed to direct the production of GdhA and GdhB with N-terminal extensions of 14 and 16 amino acid residues containing (His) ${ }_{6}$-tags, respectively, derived from the vector. To construct plasmids for the co-expression of $g d h A$ and $g d h B$ (his) genes, PCR was performed using a set of synthetic oligonucleotides, $g d h A(\mathrm{MCS} 2)-\mathrm{N} / g d h A(\mathrm{MCS} 2)-\mathrm{C}$ (Table 1), as primers and a plasmid, pETD1- $g d h A$ (his), as a template. The $g d h A$-containing fragment with the correct sequence and $g d h B$-containing fragment described above were cloned into MCS 1 and MCS 2 of pACYCDuet1, respectively. The resulting plasmid was named pACYCD1-gdhA/gdhB(his). The plasmid directed the production of $\mathrm{GdhB}$ with a (His) ${ }_{6}$-tag at the $\mathrm{N}$ terminus and GdhA without a tag. We also constructed another plasmid, pETD1-gdhA, by cloning the above $g d h A$-containing fragment into MCS 1 of pETDuet-1, which has a replication origin compatible with pACYCD1-gdhA/gdhB(his).

For overexpression of $g d h A$ (his) or $g d h B$ (his), E. coli BL21-Codon-Plus BL21(DE3)-RIL cells harbouring pETD1-gdhA(his) or $-g d h B$ (his) were cultured in $2 \times$ YT medium supplemented with $50 \mu \mathrm{g}$ kanamycin $\mathrm{ml}^{-1}$ and $30 \mu \mathrm{g}$ chloramphenicol ml $\mathrm{m}^{-1}$. When E. coli cells were grown to $\mathrm{OD}_{600} 0.6$, IPTG was added at a final concentration of $0.1 \mathrm{mM}$. Culture was continued for an additional $12 \mathrm{~h}$ after induction at $25{ }^{\circ} \mathrm{C}$. For co-expression of $g d h A$ and $g d h B($ his), E. coli BL21(DE3) cells harbouring both plasmids, pACYCD1-gdhA/gdhB(his) and pETD1-gdhA, were prepared. Since the expression level of GdhA was lower than that of GdhB(his) in E. coli cells harbouring pACYCD1$g d h A / g d h B$ (his) alone, the latter plasmid was co-introduced to enhance the expression level of GdhA. Recombinant cells were cultured by a procedure similar to that described above, although the IPTG concentration and the temperature for expression were changed to $1 \mathrm{mM}$ and $37^{\circ} \mathrm{C}$, respectively.

Purification of recombinant GdhA, GdhB and GdhA-GdhB. E. coli cells harbouring pETD1-gdhA(his) from a 1.61 culture were suspended in $32 \mathrm{ml}$ buffer I [ $20 \mathrm{mM}$ Tris/ $\mathrm{HCl}, \mathrm{pH} \mathrm{8.0,10 \% (v/v)}$ glycerol] and disrupted by sonication. The supernatant prepared by centrifugation at $40000 \mathrm{~g}$ for $15 \mathrm{~min}$ was heated at $70{ }^{\circ} \mathrm{C}$ for $30 \mathrm{~min}$, and denatured proteins from $E$. coli cells were removed by centrifugation at $40000 \mathrm{~g}$ for $15 \mathrm{~min}$. Supernatant was applied to an $\mathrm{Ni}^{2+}$-nitrilotriacetic acid (NTA) resin column (Novagen), preequilibrated with buffer I containing $20 \mathrm{mM}$ imidazole. After washing the column with a sufficient volume of buffer I containing $20 \mathrm{mM}$ 
Table 1. Oligonucleotides used in this study

Restriction sites used for cloning are underlined.

\begin{tabular}{|c|c|}
\hline Oligonucleotide & Sequence \\
\hline $\operatorname{gdhA}(\mathrm{MCS} 1)-\mathrm{N}$ & 5'-CGCCGCGGATCCGATGCCGCTAAAAGCCTAC-3' \\
\hline $\operatorname{gdhA}(\mathrm{MCS} 1)-\mathrm{C}$ & 5'-CCCCCCAAGCTTTTACGGGTACACGCCCCG-3' \\
\hline $\operatorname{gdhB}(\mathrm{MCS} 1)-\mathrm{C}$ & 5'-AAACGCGTCGACTTAAGGGTATAGGCCCCG-3' \\
\hline $\operatorname{gdhA}(\mathrm{MCS} 2)-\mathrm{N}$ & 5'-CCGCCGGAATTCCATATGCCGCTAAAAGCCTAC-3' \\
\hline $\operatorname{gdhA}(\mathrm{MCS} 2)-\mathrm{C}$ & 5'-CGGCGGGGTACCTTACGGGTACACGCCCCG-3' \\
\hline gdhBH8-C & 5'-AAACTGCAGTTAATGGTGATGGTGATGGTGATGGTGAGGGTATAGGCCCCGGAG-3' \\
\hline HTK-N & 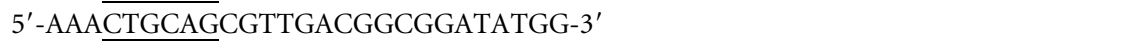 \\
\hline HTK-C & 5'-CGGGGTACCCGTAACCAACATGATTAA-3' \\
\hline TTC1210-N & $5^{\prime}$-CGGGGTACCGCTATCCCCGTGGACTGG-3' \\
\hline TTC1210-C & 5'-CCGGAATTCAGGCCCGGACCCGCCCCG-3' \\
\hline dgdhA-up-N & $5^{\prime}$-CCCCGAATTCAGATCTTCGGCCCGGTGCTT-3' \\
\hline dgdhB-dn-C & 5'-CCCCGGATCCAGTCCACCCCCGCGGAGAGG-3' \\
\hline $\mathrm{HTK}(\mathrm{KO})-\mathrm{N}$ & 5'-GGGTCTAGAGGATCTACTAGTCATATG-3' \\
\hline $\mathrm{HTK}(\mathrm{KO})-\mathrm{C}$ & 5'-GGGTCTAGACGTAACCAACATGATTAA-3' \\
\hline
\end{tabular}

imidazole, GdhA was eluted with buffer I containing $500 \mathrm{mM}$ imidazole. The purity of the recombinant protein was verified by $12 \%$ SDS-PAGE (Laemmli, 1970). Protein concentrations were determined by the Bradford method using a Bio-Rad protein assay kit (Bio-Rad) (Bradford, 1976). The recovered fractions were concentrated with a Vivaspin concentrator with a molecular weight cut-off (MWCO) of 10000 Da (Vivascience). GdhB(his) and GdhA$\mathrm{GdhB}$ (his) were also purified with a method essentially the same as that for GdhA(his). When $g d h A$ and $g d h B$ (his) were co-expressed in E. coli, GdhA and GdhB(his) were purified as (a) hetero-complex(es) at a molecular ratio of $1: 1$ on SDS-PAGE, which was estimated by ImageJ gel densitometry (Abramoff et al., 2004) of a Coomassie G250-stained gel. In all assays of the GdhA-GdhB(his) hetero-complex in this study, the GdhA-GdhB(his) preparation contained GdhA and $\mathrm{GdhB}$ (his) proteins at a $1: 1$ ratio.

GDH assay. The GDH assay was performed by the method of Ruiz et al. $(1998,2003)$ with a minor modification. In brief, the reaction mixture for oxidative deamination of Glu contained $100 \mathrm{mM}$ potassium phosphate buffer, $\mathrm{pH} 7.0,50 \mathrm{mM} \mathrm{KCl}, 50 \mathrm{mM}$ Glu and $2 \mathrm{mM} \mathrm{NAD}^{+}$. The reaction mixture for reductive amination of 2-OG contained $100 \mathrm{mM}$ potassium phosphate buffer, pH 7.0, $50 \mathrm{mM}$ $\mathrm{NH}_{4} \mathrm{Cl}, 50 \mathrm{mM} 2-\mathrm{OG}$ and $150 \mu \mathrm{M}$ NADH. After the reaction mixture was pre-heated to $60{ }^{\circ} \mathrm{C}$, the reaction was started by the addition of enzymes, and the reduction of $\mathrm{NAD}^{+}$to $\mathrm{NADH}$ or oxidation of $\mathrm{NADH}$ to $\mathrm{NAD}^{+}$was monitored at $340 \mathrm{~nm}$ using a Shimadzu UV2000 spectrophotometer. One unit of enzyme activity was defined as the amount of enzyme that formed one micromole of $\mathrm{NAD}^{+}$or NADH per minute. To examine the allosteric regulation of GDH, GDH activity was measured in both directions in the above reaction mixture supplemented with potential regulatory compounds for GDH, such as $1 \mathrm{mM}$ nucleotides (ATP, GTP, ADP and GDP), $1 \mathrm{mM}$ of various amino acids except glutamate, $500 \mu \mathrm{M} \mathrm{NADP}^{+}$or $100 \mu \mathrm{M}$ NADPH.

Steady-state kinetic analyses were carried out by measuring the decrease in absorbance in reaction mixtures containing 10-200 $\mu \mathrm{g}$ $\mathrm{ml}^{-1}$ co-purified GdhA/GdhB(his) enzyme in $100 \mathrm{mM}$ sodium phosphate buffer, $\mathrm{pH} 7.0$, at $60{ }^{\circ} \mathrm{C}$. For analyses of oxidative deamination, $50 \mathrm{mM} \mathrm{KCl}$ was added. In order to determine the $K_{\mathrm{m}}$ for $\mathrm{NADH}$, the concentration of $\mathrm{NADH}$ was varied in the range of 2$150 \mu \mathrm{M}$ using fixed concentrations of 2 -OG $(50 \mathrm{mM})$ and $\mathrm{NH}_{4} \mathrm{Cl}$ $(50 \mathrm{mM})$. In order to determine the $K_{\mathrm{m}}$ for 2-OG, the concentration of 2-OG was varied in the range of $10-5000 \mu \mathrm{M}$ using fixed concentrations of NADH $(150 \mu \mathrm{M})$ and $\mathrm{NH}_{4} \mathrm{Cl}(50 \mathrm{mM})$. In order to determine the $K_{\mathrm{m}}$ for ammonia, the concentration of $\mathrm{NH}_{4} \mathrm{Cl}$ was varied in the range of $0.5-50 \mathrm{mM}$ using fixed concentrations of 2-OG $(50 \mathrm{mM})$ and NADH $(150 \mu \mathrm{M})$. In order to determine the $K_{\mathrm{m}}$ for $\mathrm{NAD}^{+}$, the concentration of $\mathrm{NAD}^{+}$was varied in the range of $0.1-$ $10 \mathrm{mM}$ using a fixed concentration of Glu $(50 \mathrm{mM})$. In order to determine the $K_{\mathrm{m}}$ for Glu, the concentration of Glu was varied in the range of $1-50 \mathrm{mM}$ using a fixed concentration of $\mathrm{NAD}^{+}(10 \mathrm{mM})$. Leu at $1 \mathrm{mM}$ was added to the reaction mixtures when required. Most data obtained seemed to be in conformity with Michaelis-Menten kinetics; however, in the absence of $1 \mathrm{mM}$ Leu, data for Glu did not fit Michaelis-Menten kinetics but did fit the Hill equation at lower concentrations of Glu. The $K_{0.5}$ value and Hill coefficient were calculated using the Hill equation.

Preparation of GDH from T. thermophilus. Homologous recombination to obtain the T. thermophilus strain expressing His-tag-fused $g d h B$ was performed as follows. All PCR primers are listed in Table 1. The first PCR was performed with primers $g d h B \mathrm{H} 8-\mathrm{N}$ and $g d h B \mathrm{H} 8-\mathrm{C}$ to amplify $1200 \mathrm{bp}$ of the $g d h B$ gene. The latter primer was designed to add a (His) ${ }_{8}$-tag at the $\mathrm{C}$ terminus of GdhB. The second PCR was performed with primers HTK-N and HTK-C to amplify the hyperthermostable kanamycin nucleotidyltransferase (HTK) gene (Hoseki et al., 2003). The third PCR was performed with primers TTC1210-N and TTC1210-C to amplify the downstream region (1200 bp) of the $g d h B$ gene. The three amplified fragments were digested with SphI/ $P s t \mathrm{I}, P s t \mathrm{I} / K p n \mathrm{I}$ and $K p n \mathrm{I} / E c o \mathrm{RI}$, respectively, and cloned separately into pBlueScript II $\mathrm{SK}(+)$. After the DNA sequences had been verified, the three fragments were ligated together with pBlueScript II SK( + ) digested with SphI/EcoRI. The resulting plasmid, named pSKgdhBH8-HTK-1210, was used for double-crossover-type homologous 
recombination of T. thermophilus HB27 by the method of Koyama et al. (1986). Colonies growing on the TM plate supplemented with $200 \mu \mathrm{g}$ kanamycin $\mathrm{ml}^{-1}$ were selected, and recombination by double crossover in desired regions was confirmed by PCR using genomic DNA prepared from the colonies as template. Sequence analysis of the PCR-amplified fragment verified that the eight C-terminal histidine codons were correctly fused to the $g d h B$ gene on the chromosome. The strain was named T. thermophilus GdhB-H8.

T. thermophilus HB27 and GdhB-H8 cells were cultured in $1.61 \mathrm{MM}$ medium, supplemented with $50 \mu \mathrm{g}$ kanamycin $\mathrm{ml}^{-1}$ for the $T$. thermophilus B-H8 strain, at $70{ }^{\circ} \mathrm{C}$ for about $15 \mathrm{~h}$, and harvested. The cells were resuspended in $32 \mathrm{ml}$ buffer I and disrupted by sonication. Cell debris was removed by centrifugation $(40000 \mathrm{~g}$, $\left.15 \mathrm{~min}, 4{ }^{\circ} \mathrm{C}\right)$. Protease Inhibitor Cocktail $(160 \mu \mathrm{l})$ (Nacalai Tesque) was added to the supernatant. The sample was applied to an $\mathrm{Ni}^{2+}$-NTA resin column (Novagen), which was pre-equilibrated with buffer I containing $20 \mathrm{mM}$ imidazole. The column was then washed with buffer I containing $20 \mathrm{mM}$ imidazole. Adsorbed proteins were eluted with buffer I containing $500 \mathrm{mM}$ imidazole and used as purified enzymes.

Construction of $g d h A$ and $g d h B$ disruptants of $T$. thermophilus. To elucidate the cross-activity of the antibodies and metabolic role of the gene products, knockout mutants of $g d h A$ and $g d h B$ genes, and the double-knockout mutant of $T$. thermophilus lacking both $g d h A$ and $g d h B$ genes, were constructed as follows. The plasmids, named $\mathrm{pSK}-\mathrm{KO}(g d h A), \mathrm{pSK}-\mathrm{KO}(g d h B)$ and $\mathrm{pSK}-\mathrm{KO}(g d h A / g d h B)$, were used for double-crossover-type homologous recombination of $T$. thermophilus HB27 by a method of Koyama et al. (1986). All PCR primers to amplify upstream and downstream regions of the $g d h$ genes and the HTK gene are listed in Table 1. The first PCR was performed with primers dgdhA-up-N and dgdhA-up-C to amplify $1000 \mathrm{bp}$ of the upstream region of the $g d h A$ gene. The second PCR was performed with primers $\mathrm{HTK}(\mathrm{KO})-\mathrm{N}$ and $\mathrm{HTK}(\mathrm{KO})-\mathrm{C}$ to amplify the HTK gene. The third PCR was performed with primers dgdhA-dn-N and dgdhA-dn-C to amplify the downstream region (1000 bp) of the gdhA gene. The three amplified fragments prepared from the recombinant plasmids by treatment with appropriate restriction enzymes were digested with EcoRI/KpnI, XbaI and PstI/BamHI, respectively, and cloned separately into pBlueScript II SK(+). After the DNA sequences had been verified, the three fragments were ligated together with pBlueScript II SK( + ) digested with EcoRI/ $B a m H I$. The resulting plasmid, named pSK-KO $(g d h A)$, was used for homologous recombination of $T$. thermophilus HB27. Colonies growing on the TM plate supplemented with $200 \mu \mathrm{g}$ kanamycin $\mathrm{ml}^{-1}$ were selected, and recombination by double crossover in desired regions was confirmed by PCR using genomic DNA prepared from the colonies as template. pSK-KO $(g d h B)$ and $\mathrm{pSK}-\mathrm{KO}(g d h A / g d h B)$ were also constructed in a similar way and used for homologous recombination to construct the knockout mutant of the $g d h B$ gene and double-knockout mutant of both the $g d h A$ and the $g d h B$ gene.

\begin{abstract}
Western blot analysis. Rabbit polyclonal antibodies raised against GdhA(his) purified from recombinant E. coli were prepared by Scrum, Inc. For Western blot analysis, proteins were separated by $12 \%$ SDS-PAGE. After electrophoresis, proteins were transferred to a PVDF membrane (Millipore) by electroblotting. GdhA was detected using polyclonal antibodies, anti-rabbit peroxidase-linked secondary antibodies (GE Healthcare), and the ECL Plus Western blotting detection system (GE Healthcare) according to the manufacturer's instructions.
\end{abstract}

Native PAGE analysis. Native PAGE analysis was done according to the method of Goldenberg (1989) on an $8 \%(\mathrm{w} / \mathrm{v})$ polyacrylamide gel with a discontinuous buffer system containing $53 \mathrm{mM}$ Tris/ $\mathrm{HCl}$ and $68 \mathrm{mM}$ glycine $(\mathrm{pH} 8.9)$ as the cathodic buffer in the upper chamber and $100 \mathrm{mM}$ Tris/HCl $(\mathrm{pH} 7.8)$ as the anodic buffer in the lower chamber. The samples were prepared as follows. GdhA(his) and $\mathrm{GdhB}$ (his) $(15 \mu \mathrm{g}$ each) were mixed to a molar ratio of $1: 1$, and then incubated for various periods $(0,5,10,30,60$ and $120 \mathrm{~min})$ at room temperature. Separately, $15 \mu \mathrm{g}$ each of GdhA(his) and $\mathrm{GdhB}($ his $)$, and $30 \mu \mathrm{g}$ of co-expressed and co-purified GdhA-GdhB(his), which contained approximately $15 \mu \mathrm{g}$ GdhA and $15 \mu \mathrm{g}$ GdhB(his), were incubated for $120 \mathrm{~min}$ at room temperature. Leucine at $1 \mathrm{mM}$ was added, if necessary, at the start of incubation. After incubation, these samples $(30 \mu \mathrm{l})$ were mixed with a one-fourth volume $(10 \mu \mathrm{l})$ of loading buffer, which contained $40 \%(\mathrm{v} / \mathrm{v})$ glycerol, $0.4 \%$ bromophenol blue and $200 \mathrm{mM}$ Tris/ $\mathrm{HCl}$ (pH 6.8), and subjected to PAGE. After appropriate electrophoresis, the gel was stained with Coomassie G-250.

\section{RESULTS}

\section{GDH activities of recombinant GdhA and GdhB}

T. thermophilus HB27 has two gdh genes, gdhA (TTC1212) and $g d h B$ (TTC1211), which are juxtaposed in the same direction on the genome. Both $g d h$ homologues encode proteins classified as GDH50s, sharing $46 \%$ identity in the amino acid sequence. GdhB shows high sequence similarity to the putative GdhB orthologues TTHA1576 (putative GdhB) from T. thermophilus HB8 (99\% identity), putative GDH from Thermus aquaticus (91\% identity) and Dgeo_0494 from Deinococcus geothermalis DSM11300 (70\% identity). GdhB also shows relatively high similarity to homooligomeric GDH50s from Thermotoga maritima (TmGDH), Thermococcus profundus (TpGDH), Pyrococcus islandicum (PiGDH), humans and cattle (56, 50, 48, 43 and $43 \%$ identity, respectively). In contrast, although GdhA exhibits high similarity to the putative GdhA homologues TTHA1577 (putative GdhA) from T. thermophilus HB8 (98\% identity), putative GDH from $T$. aquaticus (78\% identity) and Dgeo_0945 from D. geothermalis DSM11300 (48\% identity), GdhA shows somewhat lower similarity than GdhB to its homologues TmGDH, TpGDH, PiGDH, and GDHs from humans and cattle, of $45,42,38,34$ and $34 \%$, respectively. When evolutionary relationships among GdhA, GdhB and related GDHs were analysed, the phylogenetic tree showed that GdhB from T. thermophilus HB27 forms a lineage with homo-oligomeric GDHs including TmGDH (Supplementary Fig. S1). On the other hand, GdhA, TTHA1577, putative GdhA from T. aquaticus and Dgeo_0495 are found in a separate lineage, suggesting that they have evolved to have functions different from those of GDH species that contain GdhB. GdhA and GdhB are composed of 419 and 424 amino acid residues, respectively, and their estimated molecular masses are 44.5 and $46.0 \mathrm{kDa}$. To characterize GdhA and $\mathrm{GdhB}$, the proteins were purified from recombinant E. coli cells in the His-tag-fused form (Fig. 2a, b). GdhA(his) has no activity in reductive amination and oxidative deamination reactions (Fig. 3). Moreover, no dehydrogenase activity of GdhA(his) was detected when similar compounds, $\alpha$ aminoadipate and aspartate, and leucine were used as substrates (data not shown). Consistent with this, although GdhA contains most amino acid residues important for catalytic function, Ala was substituted for Lys113 (Fig. 4), which is the catalytic residue of GDH that interacts with the 
(a)

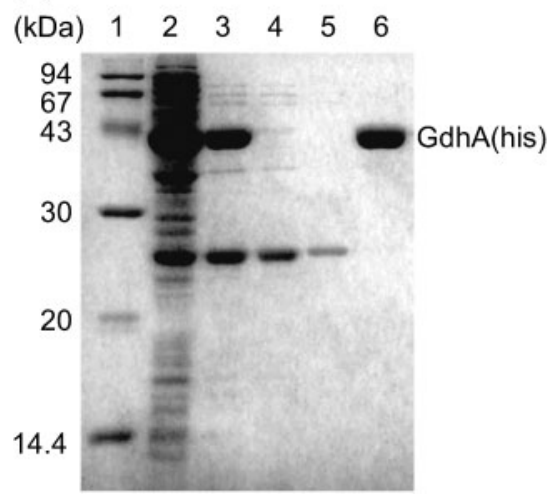

(b)

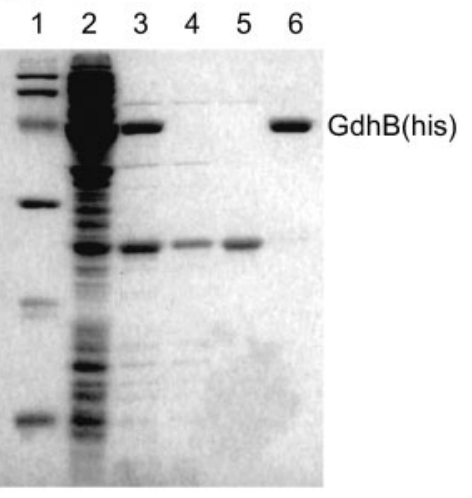

(c)

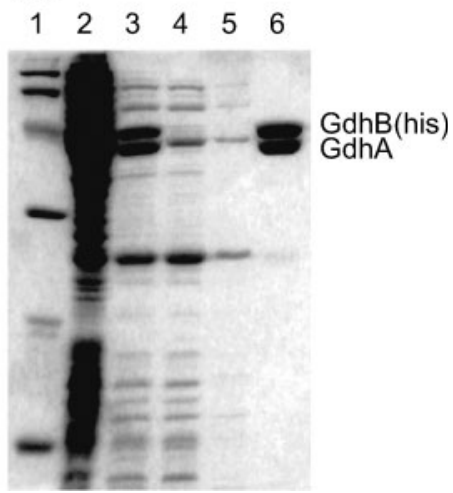

Fig. 2. Purification of recombinant GdhA(his) (a), GdhB(his) (b) and co-expressed GdhA-GdhB(his) (c) analysed by SDSPAGE. Lanes: 1, molecular size marker; 2, crude extract of $E$. coli cells; 3, supernatants after heat treatment; 4, passing fractions of $\mathrm{Ni}^{2+}$-affinity chromatography; 5 , washing fractions; 6 , fractions eluted by $500 \mathrm{mM}$ imidazole.

-amino group of glutamate (Baker et al., 1992; Stillman et al., 1993) in GdhA. GdhB(his) was found to exhibit a specific activity of $12.7 \mathrm{U} \mathrm{mg}^{-1}$ for reductive amination of $2-\mathrm{OG}$ and $9.5 \mathrm{U} \mathrm{mg}^{-1}$ for oxidative deamination of glutamate (Fig. 3).

\section{Interaction of GdhA with GdhB}

To examine the possible interaction of GdhA with GdhB, we measured the GDH activity of GdhB(his) in the presence of various amounts of $\mathrm{GdhA}$ (his). $\mathrm{GdhB}$ (his) was mixed with various amounts of GdhA(his) and the GDH activity of $\mathrm{GdhB}$ (his) was measured after $10 \mathrm{~min}$ incubation at room

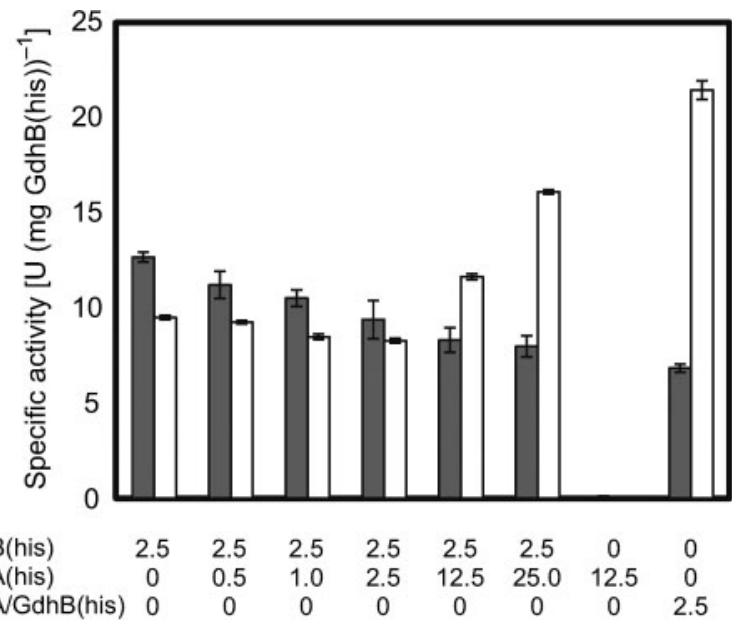

Fig. 3. GDH activities of recombinant enzymes. Grey and white bars indicate the specific activities per milligram $\mathrm{GdhB}(\mathrm{his})$ for reductive amination of $2-\mathrm{OG}$ and oxidative deamination of glutamate, respectively. The amounts of protein $(\mu \mathrm{g})$ added to the reaction are shown below the bars. The amount of protein, $2.5 \mu \mathrm{g}$, in GdhA-GdhB(his) denotes the amount of GdhB(his) in the GdhA-GdhB(his) complex. temperature. As shown above, $\mathrm{GdhB}$ (his) shows $12.7 \mathrm{U}$ [mg $\mathrm{GdhB}$ (his) $]^{-1}$ for reductive amination of $2-\mathrm{OG}$ in the absence of $\mathrm{GdhA}$ (his). Interestingly, the reductive amination activity of $\mathrm{GdhB}$ (his) decreased with the increase in the amount of GdhA(his) added to the reaction mixture, and when $\mathrm{GdhA}$ (his) was added to the mixture at a ratio of $10: 1$, activity decreased to $8.0 \mathrm{U} \mathrm{mg}^{-1} \mathrm{GdhB}$ (his) (Fig. 3). On the other hand, the oxidative deamination activity towards glutamate of $\mathrm{GdhB}($ his $)\left\{9.5 \mathrm{U}[\mathrm{mg} \mathrm{GdhB}(\text { his })]^{-1}\right\}$ was increased to $16.1 \mathrm{U}$ [mg GdhB(his) ${ }^{-1}$ when $\mathrm{GdhA}$ (his) was added to the mixture at a ratio of $1: 10$. To examine the direct protein-protein interaction between GdhA and GdhB, GdhA without a tag was co-expressed with $\mathrm{GdhB}$ (his) in E. coli cells. When the heat-stable fraction of crude extract was applied to $\mathrm{Ni}^{2+}$-affinity chromatography, GdhA was co-purified with $\mathrm{GdhB}$ (his) (Fig. 2c), indicating hetero-oligomer formation between GdhA and GdhB(his). Densitometric analysis of the Coomassie G-250-stained SDS-PAGE gel by ImageJ revealed that the purified fraction contained GdhA and $\mathrm{GdhB}$ (his) at a molecular ratio of $1: 1$. GdhA-GdhB(his) hetero-oligomer co-purified at a ratio of $1: 1$ showed an activity of $6.8 \mathrm{U}[\mathrm{mg} \operatorname{GdhB}(\text { his })]^{-1}$ for reductive amination of $2-\mathrm{OG}$ and $21.5 \mathrm{U}[\mathrm{mg} \mathrm{GdhB}(\mathrm{his})]^{-1}$ for oxidative deamination of glutamate in an $\mathrm{NAD}(\mathrm{H})$ dependent manner (Fig. 3). These results suggest that changes in both the specific activity and preference of the reaction direction of $\mathrm{GdhB}$ (his) by the addition of GdhA(his) were caused by the interaction of GdhA with $\mathrm{GdhB}$ (his). When $\mathrm{NADP}(\mathrm{H})$ was used, the specific activity of co-purified GdhA-GdhB(his) hetero-oligomer was decreased to one-third for both reactions (data not shown), indicating the $\mathrm{NAD}(\mathrm{H})$ preference of this $\mathrm{GDH}$.

\section{Allosteric activation of GdhA-GdhB by amino acids}

The activity of mammalian GDH is known to be regulated allosterically by small molecules, such as nucleotides and 

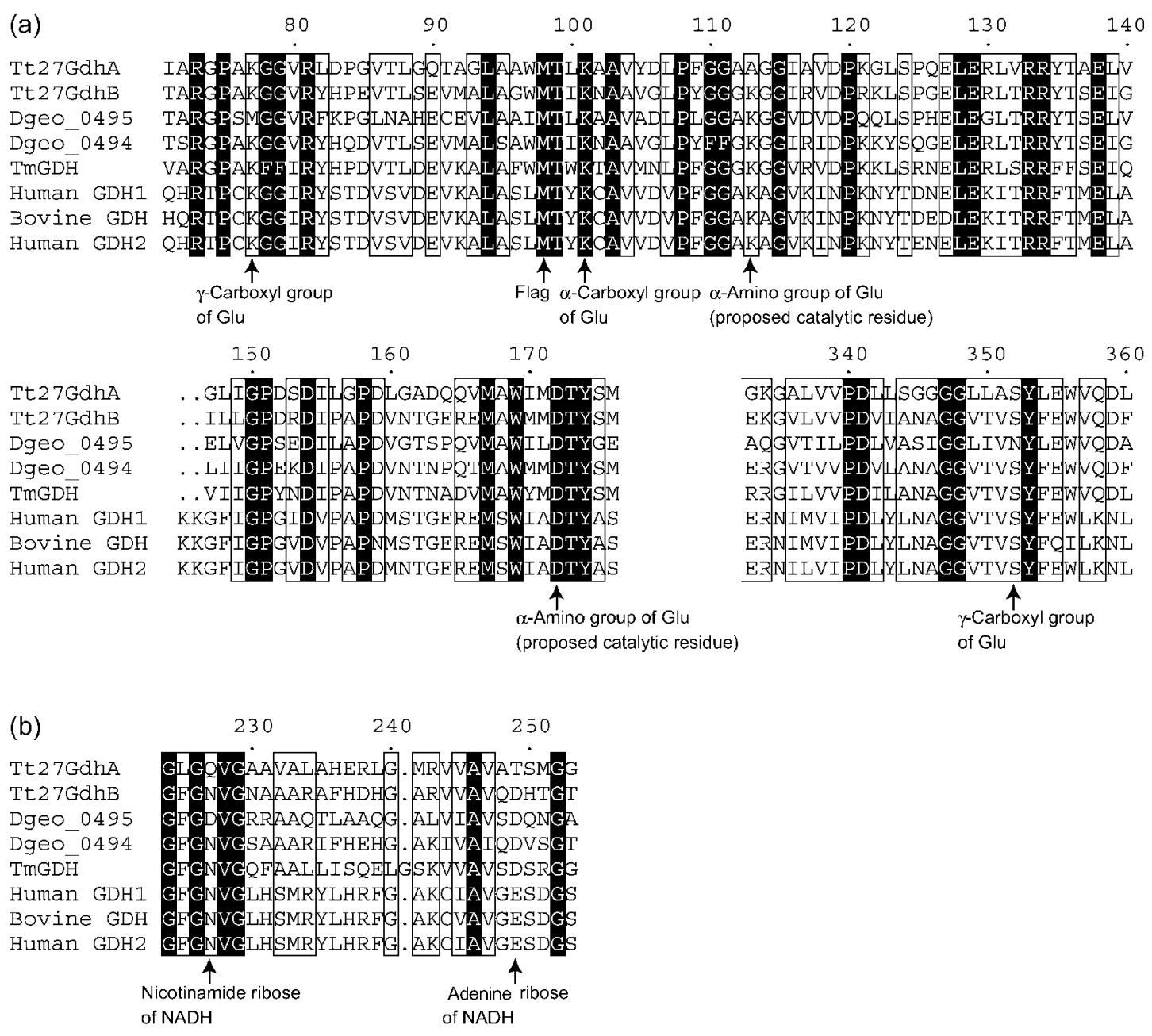

Fig. 4. Multiple amino acid sequence alignment of GDH. Amino acid sequences were aligned using CLUSTAL W (Thompson et al., 1994). The numbers above the alignment are the residue numbers of GdhA from T. thermophilus HB27. Roles in catalysis and substrate/coenzyme recognition of several residues that are proposed on the basis of crystal structures of other GDHs are indicated by arrows. Tt27GdhA and Tt27GdhB represent GdhA and GdhB from T. thermophilus HB27, respectively. Dgeo_0495 and Dgeo_0494 indicate putative GdhA and GdhB homologues from D. geothermalis DSM11300, respectively. TmGDH stands for GDH from T. maritima. (a) Amino acid sequences around glutamate-recognizing residues in TmGDH. (b) Amino acid sequences around $\mathrm{NADH}$-recognizing residues in $\mathrm{TmGDH}$.

amino acids. We therefore examined whether small molecules influence the activity of recombinant GdhAGdhB(his) co-purified from E. coli cells. When several compounds were added at $1 \mathrm{mM}$, GDH activity was increased, especially by the addition of branched-chain amino acids, hydrophobic amino acids and aromatic amino acids (Table 2). Among them, leucine exhibited the highest activation in both directions. The resulting activities were $974 \%$ for reductive amination and $245 \%$ for oxidative deamination. Other amino acids, most of which are hydrophilic amino acids, modulated GDH activity slightly. In contrast, the GDH activity of $\mathrm{GdhB}$ (his) alone was not greatly affected by these compounds, although moderate activation by leucine $(151 \%)$ was observed in oxidative deamination. These results suggest that GdhA, which possesses no activity, mediates the allosteric activation of GdhB.

\section{Effects of leucine on GDH activity of co-purified GdhA-GdhB}

In the following analyses, we chose leucine as an effector to examine the effect of amino acid addition on GDH activity, and added leucine to the co-purified GdhA-GdhB(his) assay mixture at various concentrations (Fig. 5). The oxidative deamination activity of GdhA-GdhB(his) was threefold higher than that of reductive amination without leucine. As the leucine concentration increased, both activities were increased; however, reductive amination activity was influenced to a greater extent than oxidative 
Table 2. Effects of possible effectors on GDH activities

ND, Not determined; AAA, $\alpha$-aminoadipate.

\begin{tabular}{|c|c|c|c|c|}
\hline \multirow[t]{2}{*}{ Compound } & \multicolumn{2}{|c|}{ GdhA-GdhB(his) } & \multicolumn{2}{|c|}{ GdhB(his) } \\
\hline & Reductive amination (\%) & Oxidative deamination (\%) & Reductive amination (\%) & Oxidative deamination (\%) \\
\hline Leu & $974 \pm 47$ & $245 \pm 12$ & $96 \pm 2$ & $151 \pm 1$ \\
\hline Met & $772 \pm 31$ & $238 \pm 8$ & $\mathrm{ND}$ & ND \\
\hline Val & $604 \pm 41$ & $205 \pm 7$ & ND & ND \\
\hline Tyr & $446 \pm 9$ & $150 \pm 2$ & ND & ND \\
\hline Phe & $437 \pm 14$ & $145 \pm 1$ & ND & ND \\
\hline His & $315 \pm 15$ & $123 \pm 2$ & ND & ND \\
\hline $\operatorname{Trp}$ & $123 \pm 3$ & $96 \pm 1$ & ND & ND \\
\hline Cys & $184 \pm 18$ & $140 \pm 3$ & $\mathrm{ND}$ & $\mathrm{ND}$ \\
\hline Arg & $174 \pm 1$ & $106 \pm 5$ & $91 \pm 5$ & $101 \pm 1$ \\
\hline Asn & $142 \pm 2$ & $99 \pm 3$ & ND & ND \\
\hline Thr & $127 \pm 3$ & $99 \pm 2$ & $\mathrm{ND}$ & ND \\
\hline Ser & $114 \pm 5$ & $96 \pm 2$ & $\mathrm{ND}$ & ND \\
\hline Ala & $113 \pm 4$ & $96 \pm 3$ & $\mathrm{ND}$ & $\mathrm{ND}$ \\
\hline Gly & $115 \pm 6$ & $95 \pm 2$ & $\mathrm{ND}$ & ND \\
\hline AAA & $134 \pm 11$ & $99 \pm 3$ & ND & ND \\
\hline NADPH & $64 \pm 18$ & $77 \pm 2$ & $82 \pm 18$ & $97 \pm 1$ \\
\hline $\mathrm{NADP}^{+}$ & $82 \pm 6$ & $64 \pm 0$ & $89 \pm 10$ & $105 \pm 1$ \\
\hline GTP & $89 \pm 6$ & $99 \pm 1$ & $88 \pm 9$ & $92 \pm 2$ \\
\hline GDP & $93 \pm 8$ & $101 \pm 4$ & $94 \pm 12$ & $100 \pm 2$ \\
\hline ATP & $107 \pm 7$ & $101 \pm 0$ & $83 \pm 9$ & $95 \pm 1$ \\
\hline
\end{tabular}

${ }^{\star} \mathrm{A}$ value of $100 \%$ denotes activities of 6.8 and $21.5 \mathrm{U}[\mathrm{mg} \mathrm{GdhB}(\mathrm{his})]^{-1}$ for reductive amination and oxidative deamination, respectively. $\dagger \mathrm{A}$ value of $100 \%$ denotes activities of 12.7 and $9.5 \mathrm{U}$ [mg GdhB(his) $]^{-1}$ for reductive amination and oxidative deamination, respectively.

deamination activity. In the presence of $10 \mathrm{mM}$ leucine, reductive amination activity became 2.1-fold higher than oxidative deamination activity.

To further examine the mechanism of GdhA-GdhB activation by leucine, we determined the kinetic parameters of GdhA-GdhB(his) in the presence and absence of $1 \mathrm{mM}$ leucine (Table 3). In the absence of leucine, GdhAGdhB(his) exhibited low apparent $K_{\mathrm{m}}$ values for NADH and 2-OG of 4.8 and $27 \mu \mathrm{M}$, respectively, and an apparent $K_{\mathrm{m}}$ value for $\mathrm{NH}_{4}^{+}$of $3500 \mu \mathrm{M}$, which is remarkably low compared with those for many other GDHs. When leucine was added to the reaction mixture, the apparent $K_{\mathrm{m}}$ values for $\mathrm{NADH}$ and 2-OG were decreased by 1.7- and 2.5-fold, respectively, while the apparent $K_{\mathrm{m}}$ value for ammonia was increased by 4.8 -fold. A marked change was also observed in the $k_{\text {cat }}$ value for the reaction, which exhibited a maximal 11-fold increase. In oxidative deamination, in the range of 1-50 $\mathrm{mM}$ Glu, data fitted Michaelis-Menten kinetics. GdhA-GdhB(his) exhibited higher apparent $K_{\mathrm{m}}$ values for $\mathrm{NAD}^{+}$and Glu of 150 and $4200 \mu \mathrm{M}$, respectively. When leucine was added to the reaction mixture, the apparent $K_{\mathrm{m}}$ value for Glu decreased by 3.6fold, while the apparent $K_{\mathrm{m}}$ value for $\mathrm{NAD}^{+}$was slightly increased by 1.2 -fold with a twofold increase of the $k_{\text {cat }}$ value. However, in the absence of $1 \mathrm{mM}$ Leu, data did not fit the Michaelis-Menten model but did fit the Hill equation at lower concentrations of Glu (Supplementary Fig. S2). The $K_{0.5}$ value and Hill coefficient were calculated to be $20 \mu \mathrm{M}$ and 0.5 , respectively, suggesting that GdhA$\mathrm{GdhB}$ (his) showed negative cooperativity for Glu. Neither negative nor positive cooperativity for Glu was observed in the presence of $1 \mathrm{mM}$ Leu. In the reductive amination reactions, cooperativity was not observed for 2-OG irrespective of the presence or absence of $1 \mathrm{mM}$ Leu.

\section{Hetero-complex formation between GdhA and GdhB in T. thermophilus}

To verify the hetero-complex formation of GDH in $T$. thermophilus, we used anti-GdhA(his) antibodies. We also 


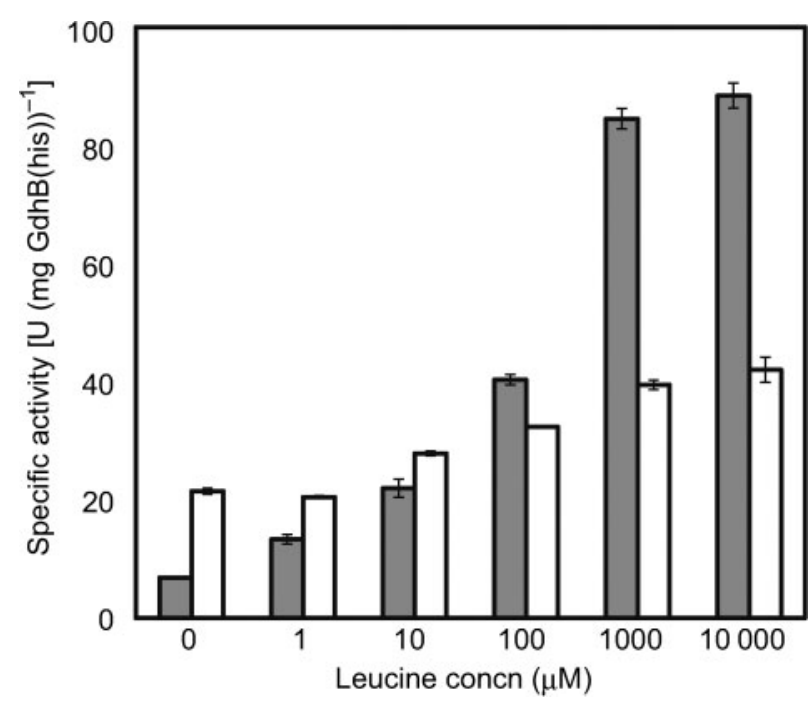

Fig. 5. Activity profile of $\mathrm{GdhA}-\mathrm{Gdh} B($ his $)$ at various leucine concentrations. Grey and white bars indicate, respectively, the specific activities for reductive amination and oxidative deamination per milligram GdhB(his).

constructed three knockout mutants, $\Delta g d h A, \Delta g d h B$ and $\Delta g d h A / \Delta g d h B$, of $T$. thermophilus. When cell lysates prepared from these mutants were analysed by Western blotting using anti-GdhA antibodies, a GdhA band reactive with anti-GdhA(his) antibodies was seen for cell lysate of the wild-type strain and the $\Delta g d h B$ mutant, whereas in the cell lysate of the knockout mutants lacking the $g d h A$ gene or both the $g d h A$ and the $g d h B$ gene, the corresponding band was missing (Fig. 6a, b). These results indicate that the antibodies specifically discriminate GdhA from other proteins. Consistent with this, the antibodies specifically recognized GdhA but not GdhB (Fig. 6c). Then, to examine whether hetero-complex formation between GdhA and GdhB occurs in $T$. thermophilus cells, an $\mathrm{Ni}^{2+}$-affinity pulldown assay of GdhA was conducted using GdhB with a (His) ${ }_{8}$-tag at the $\mathrm{C}$ terminus. Cell lysate of recombinant $T$. thermophilus GdhB-H8, which was constructed to produce
GdhB with a C-terminal (His) $)_{8}$-tag and GdhA without tag, was applied to an $\mathrm{Ni}^{2+}$-affinity column, and Western blotting analysis was conducted for the whole-cell lysate and the adsorbed fraction using anti-GdhA(his). When the cell lysate from wild-type strain T. thermophilus HB27 was used, GdhA visualized by anti-GdhA(his) antibodies was detected in the whole-cell lysate (Fig. 6d, lane 2), but no protein recognized by anti-GdhA antibodies was detected in the fraction adsorbed to the $\mathrm{Ni}^{2+}$-affinity column (Fig. $6 \mathrm{~d}$, lane 4). On the other hand, GdhA was detected in the adsorbed fraction when the lysate of T. thermophilus GdhBH8 cells was used (Fig. 6d, lanes 1 and 3 ). This result clearly indicates hetero-complex formation between GdhA and GdhB in T. thermophilus cells.

\section{Analysis of GdhA-GdhB hybrids by native gel electrophoresis}

To analyse further the hybrid formation between GdhA and GdhB, we conducted native PAGE analysis of GdhAGdhB(his) co-purified from E. coli cells (Fig. 7). The copurified GdhA-GdhB(his) migrated in the gel mainly as two separate bands. To identify each band, purified GdhA(his), $\mathrm{GdhB}$ (his) and mixtures of purified GdhA(his) and $\mathrm{GdhB}$ (his) were applied to the gel. In the native gel electrophoresis, GdhA(his) and GdhB(his) behaved differently. GdhA(his) monomer (46.5 kDa) had a calculated isoelectric point of 5.1, while $\mathrm{GdhB}$ (his) monomer $(48.0 \mathrm{kDa})$ had a calculated isoelectric point of 6.7. According to the difference in isoelectric point, GdhA(his) moved faster than GdhB(his). When GdhA(his) and GdhB(his) were applied to the gel immediately after mixing (Fig. 7, lane 4), two bands corresponding to GdhA(his) and $\mathrm{GdhB}$ (his) were observed as major species. In contrast, new two bands emerged between the parent bands at regular intervals after incubation for longer than $5 \mathrm{~min}$. This result indicates that GdhA(his) and $\operatorname{GdhB}$ (his) form hybrids at $1: 2$ and $2: 1$ ratios. From these observations, we were able to conclude that the two separate bands seen for the copurified GdhA-GdhB(his) correspond to $1: 2$ and $2: 1$ hybrids of GdhA-GdhB(his). GDH50s are known to form

Table 3. Kinetic parameters for GdhA-GdhB(his)

\begin{tabular}{|c|c|c|c|c|}
\hline \multirow[t]{2}{*}{ Substrate } & \multicolumn{2}{|c|}{ Absence of Leu } & \multicolumn{2}{|c|}{ Presence of $1 \mathrm{mM}$ Leu } \\
\hline & $k_{\mathrm{m}}^{\mathrm{app}}(\mu \mathrm{M})$ & $k_{\text {cat }}^{\text {app }}\left(\mathrm{s}^{-1}\right)$ & $k_{\mathrm{m}}^{\mathrm{app}}(\mu \mathrm{M})$ & $k_{\text {cat }}^{\text {acp }}\left(\mathrm{s}^{-1}\right)$ \\
\hline \multicolumn{5}{|c|}{ Reductive amination } \\
\hline NADH & $4.8 \pm 1.0$ & $7.1 \pm 0.4$ & $2.9 \pm 0.1$ & $47 \pm 0.3$ \\
\hline $2-\mathrm{OG}$ & $27 \pm 0.1$ & $5.8 \pm 0.0$ & $11 \pm 1.5$ & $30 \pm 1.5$ \\
\hline $\mathrm{NH}_{4}^{+}$ & $3500 \pm 120$ & $5.0 \pm 0.5$ & $17000 \pm 3600$ & $55 \pm 5.0$ \\
\hline \multicolumn{5}{|c|}{ Oxidative deamination } \\
\hline $\mathrm{NAD}^{+}$ & $150 \pm 30$ & $17 \pm 0.7$ & $180 \pm 22$ & $33 \pm 0.8$ \\
\hline Glu & $4200 \pm 930^{*}$ & $16 \pm 1.2^{*}$ & $1200 \pm 240$ & $32 \pm 1.3$ \\
\hline
\end{tabular}

${ }^{\star}$ These values were calculated by the Michaelis-Menten model using data obtained with Glu concentrations of $1-50 \mathrm{mM}$. 
(a)

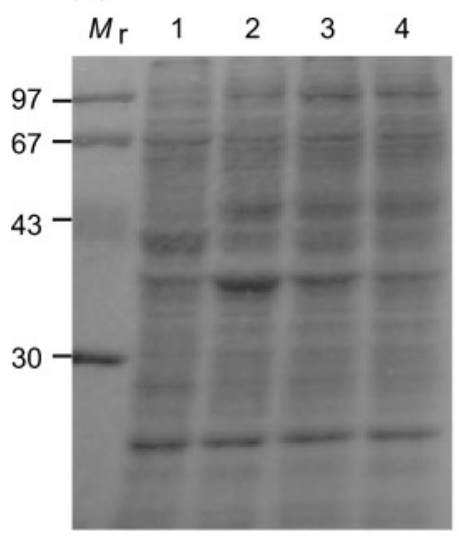

(b)

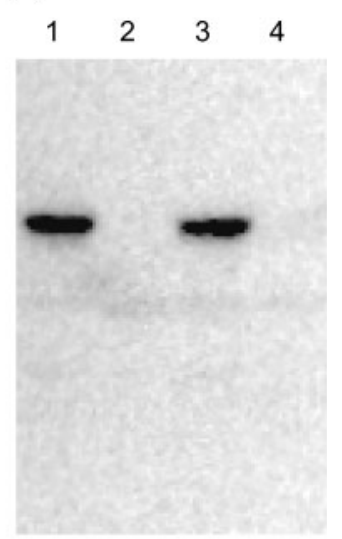

(c)

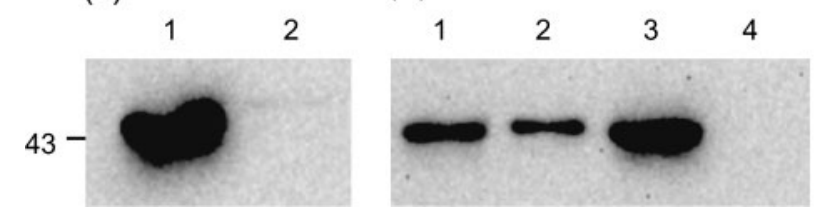

Fig. 6. Western blotting analysis using anti-GdhA antibodies. (a) SDS-PAGE (12\%) of whole-cell lysate. Lanes: 1, T. thermophilus HB27 (wild-type); 2, T. thermophilus HB27 $\Delta g d h A ; 3, T$. thermophilus HB27 $\Delta g d h B ; 4, T$. thermophilus HB27 $\Delta g d h A$ / $\Delta g d h B$. (b) Western blotting using anti-GdhA(his) antibodies for cell lysates. Lanes are the same as in (a). (c) Western blotting using anti-GdhA(his) antibodies for recombinant GdhA(his) (lane 1) and GdhB(his) (lane 2). Samples (50 ng per lane) of purified proteins were applied. (d) Lanes 1 and 3, cell lysates of $T$. thermophilus GdhB-H8 and HB27, respectively; lanes 2 and 4, $\mathrm{Ni}^{2+}$-affinity column-adsorbed fractions of GdhB-H8 and HB27, respectively.

hexamers. Therefore, we presume that either GdhB or GdhA alone forms a homo-hexamer, while the 1:2 GdhA-GdhB hybrid enzyme consists of two GdhA subunits and four GdhB subunits, and the 2:1 hybrid enzyme contains four
GdhA subunits and two GdhB subunits. Thus, the purified fraction containing GdhA and $\mathrm{GdhB}$ (his) at an apparent molecular ratio of $1: 1$ was shown to be a mixture of $\mathrm{GdhA}_{4} /$ $\mathrm{GdhB}(\text { his })_{2}$ and $\mathrm{GdhA}_{2} / \mathrm{GdhB}(\text { his })_{4}$.

\section{In vivo function of GdhAB}

In some micro-organisms, GDH is required for rapid removal of a high concentration of glutamate, while in other micro-organisms it also serves as a primary enzyme for ammonium assimilation. To elucidate the in vivo role of GdhA and GdhB in T. thermophilus, we examined the growth of T. thermophilus mutants lacking either the $g d h A$ or the $g d h B$ gene and the mutant lacking both genes. For growth analysis, we used two different liquid media, $\mathrm{MM}$ containing $14.6 \mathrm{mM}$ sucrose and $18.9 \mathrm{mM} \mathrm{NH}_{4} \mathrm{Cl}$ as $\mathrm{C}$ and $\mathrm{N}$ sources, respectively, and $\mathrm{E}$ medium containing $5 \mathrm{mM}$ Glu as both $\mathrm{C}$ and $\mathrm{N}$ source instead of sucrose and $\mathrm{NH}_{4} \mathrm{Cl}$ in MM. Wild-type, $\Delta g d h A, \Delta g d h B$ and $\Delta g d h A / \Delta g d h B$ strains grew in $M M$ at a similar growth rate (Fig. $8 \mathrm{a}$ ). This result suggests that neither GdhA nor GdhB contributes to ammonium assimilation under these conditions. On the other hand, $\Delta g d h B$ and $\Delta g d h A / \Delta g d h B$ strains did not grow in $\mathrm{E}$ medium at all (Fig. 8b). This observation clearly indicates that GdhB is required for glutamate degradation. In general, enzymes involved in catabolism prefer $\mathrm{NAD}^{+}$to $\mathrm{NADP}^{+}$. The observation that $\mathrm{NAD}^{+}$-dependent GdhB is involved in glutamate degradation may be consistent with the coenzyme specificity of $\mathrm{GdhB}$, which has a preference for $\mathrm{NAD}^{+}$over NADP ${ }^{+}$. In E medium, the $\triangle g d h A$ strain grew more slowly than $g d h A^{+}$ strains, although the $\Delta g d h A$ strain still possesses the $g d h B$ gene, suggesting a role for GdhA in Glu degradation.

\section{DISCUSSION}

Native GDH from T. thermophilus HB8 was partially purified and characterized by Ruiz et al. $(1998,2003)$. The purified GDH preparation contained two major bands that showed similar mobility on SDS-PAGE. The authors

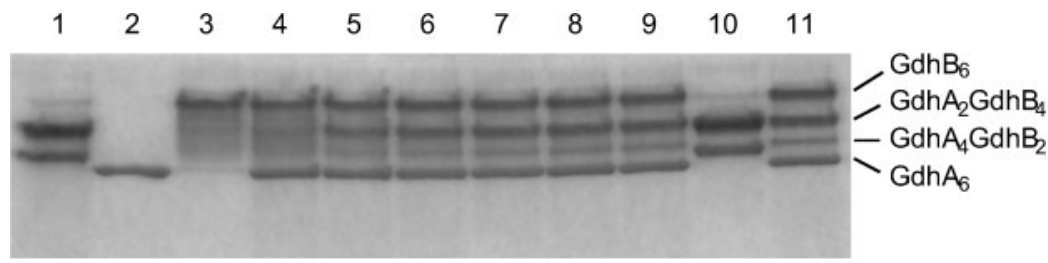

Fig. 7. Native PAGE analyses of GdhA and GdhB. Lanes: 1, co-expressed GdhAGdhB(his); 2, GdhA(his); 3, GdhB(his); 4-9, $1: 1$ mixture of $\mathrm{GdhA}($ his) and $\mathrm{GdhB}($ his). GdhA(his) and GdhB(his) expressed and purified separately were mixed and incubated for appropriate periods. After incubation, samples were applied to the native gel. Incubation times are: lane 4, $0 \mathrm{~min}$; lane 5, $5 \mathrm{~min}$; lane 6, $10 \mathrm{~min}$; lane 7, $30 \mathrm{~min}$; lane 8, $60 \mathrm{~min}$; lane 9, $120 \mathrm{~min}$. Lane 10, co-expressed GdhA$\mathrm{GdhB}$ (his) incubated for 10 min after adding $1 \mathrm{mM}$ leucine; lane 11, 1:1 mixture of $\mathrm{GdhA}($ his) and $\mathrm{GdhB}(\mathrm{his})$ incubated for $10 \mathrm{~min}$ after adding $1 \mathrm{mM}$ leucine. 
(a)

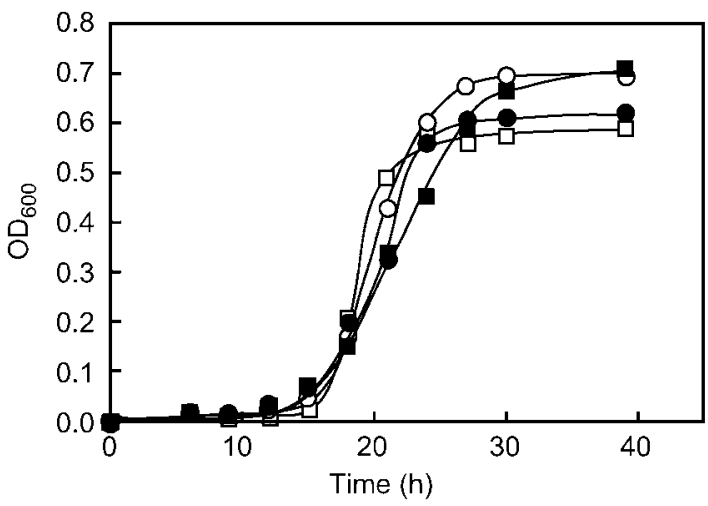

(b)

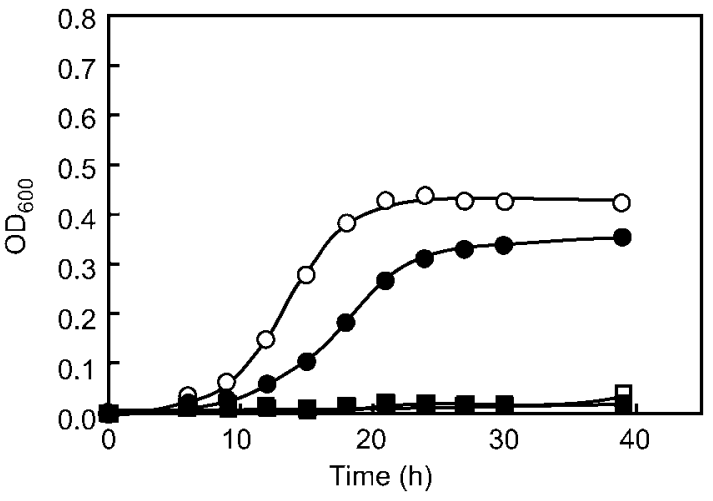

Fig. 8. Growth of wild-type $T$. thermophilus and the knockout mutants $\Delta g d h A, \Delta g d h B$ and $\Delta g d h A / \Delta g d h B$. Growth curve of wild-type T. thermophilus ( $\bigcirc), \Delta g d h A(\bullet), \Delta g d h B(\square)$ and $\Delta g d h A / \Delta g d h B(\boldsymbol{\square})$ in MM medium (a) and in E medium (b). Each strain was grown in three separate test tubes for each growth test and typical growth curves with mean values are shown.

suggested that the band of $50 \mathrm{kDa}$ corresponded to native $\mathrm{GDH}$ of this micro-organism and that another band of $48 \mathrm{kDa}$ showing faster migration on SDS-PAGE might be a partially degraded product of the upper band. Later, genome sequences were determined for both T. thermophilus HB27 and HB8. The analysis showed that both strains contain two putative GDH genes: $g d h B(T T C 1211)$ and $g d h A(T T C 1212)$ for HB27, and TTHA1576 and TTHA1577 for HB8, which putatively form an operon on the genome. TTC1211/ TTHA1576 and TTC1212/TTHA1577 are composed of 424 and 419 amino acid residues, respectively, and the molecular masses of a single subunit are calculated to be 46.0 and $44.5 \mathrm{kDa}$, respectively; therefore, we assume that the two bands reported by Ruiz and colleagues represent GdhB and GdhA of $T$. thermophilus HB8. Recently, Bolivar and colleagues reported the successful stabilization of TTC1211 produced in E. coli by immobilization (Bolivar et al., 2008); however, detailed enzymic characterization was not performed. Here, we characterized both GdhB (TTC1211) and GdhA (TTC1212) in detail, and found that GDH functions as a hetero-oligomer, where GdhB and GdhA function as catalytic and regulatory subunits, respectively. Crystal structures have been determined for GDH from $C$. symbiosum. The structural analysis revealed the roles of amino acid residues in catalytic function (Baker et al., 1992; Stillman et al., 1993). In GdhA, most residues important for catalytic functions, including the Ser (Ser352 in GdhA) and Lys (Lys77 in GdhA) residues involved in the recognition of the $\gamma$-carboxyl group of glutamate and the catalytic aspartate residue (Asp153 in GdhA), are conserved; however, Ala was substituted for Lys113 (Fig. 4), which is the catalytic residue of GDH that interacts with the $\alpha$-amino group of glutamate (Baker et al., 1992; Stillman et al., 1993). In addition, residues Asn and Asp conserved for coenzyme binding are replaced with Gln (Gln228 in GdhA) and Thr (Thr249 in GdhA), respectively, in GdhA (Fig. 4), suggesting a decreased affinity for the enzyme. All these facts coincide well with our present results, in which GdhA had no activity towards not only Glu but also other related compounds. Interestingly, $D$. geothermalis, which is related to $T$. thermophilus taxonomically, possesses two $g d h$ homologues, Dgeo_0494 and Dgeo_0495, which are juxtaposed on the genome. Dgeo_0494 is similar to GdhB, while Dgeo_0495 is similar to GdhA in amino acid sequence (Fig. 4 and Supplementary Fig. S1). In Dgeo_0495, a lysine and a serine residue, both of which bind the $\gamma$-carboxyl group of bound glutamate, and an asparagine residue for binding nicotinamide ribose of $\mathrm{NADH}$, are replaced, suggesting that Dgeo_0495 is inactive, like GdhA in T. thermophilus. Although amino acid replacements are introduced into different positions between Dgeo_0495 and GdhA, similar hetero-oligomeric GDH formation in D. geothermalis is postulated.

When GdhA(his) was added to the reaction mixture for GdhB(his), a significant decrease in reductive amination activity and a significant increase in oxidative deamination activity were observed, and a co-expressed GdhA$\mathrm{GdhB}$ (his) preparation containing each molecule at a $1: 1$ ratio showed a distinct preference for oxidative deamination to reductive amination. Furthermore, when $g d h A$ and $g d h B$ (his) were co-expressed in recombinant E. coli and $T$. thermophilus, GdhA and $\mathrm{GdhB}$ (his) were co-purified in both types of cell, implying hetero-complex formation between GdhA and GdhB. All these observations indicate that GdhA affects the activity of GdhB through heterocomplex formation. On the other hand, GdhA-GdhB(his) was activated by several amino acids, while the activity of GdhB alone was not so affected. This result indicates that hetero-complex formation between GdhA and GdhB is critical for allosteric regulation. Native hetero-complexes of GDH are known in plants. In Arabidopsis thaliana, NADGDH1 and NAD-GDH2 form hetero-hexamers composed of $\alpha$ and $\beta$ subunits at different ratios, depending on the relative expression level of each GDH subunit in various tissues (Turano et al., 1997); however, the roles of plant 
hetero-complex formation are not well understood because every GDH hexamer has almost the same activity. A hetero-complex of GDH was seen between co-expressed $E$. coli and Klebsiella aerogenes GDHs (Mountain et al., 1985) and between $a m^{1}$ and $a m^{3}$ GDHs of N. crassa (Watson \& Wootton, 1978). In contrast, hetero-oligomeric GDH from T. thermophilus is unique because only GdhB shows activity, while GdhA itself is inactive and involved in an allosteric activation mechanism. Similar functional differentiation through hetero-complex formation between paralogues is found in isocitrate dehydrogenase (IDH) from Saccharomyces cerevisiae (Barnes et al., 1971). In S. cerevisiae, IDH1 functions as the catalytic subunit and IDH2 functions as the regulatory subunit by forming an $\alpha_{4} \beta_{4}$-type hetero-octameric structure (Gabriel et al., 1986). In that system, hetero-complex formation has the advantage of allowing growth in acetate medium $(\mathrm{Hu}$ et al., 2006). Although IDH2 shares $42 \%$ amino acid sequence identity with IDH1, it shows the replacement of two residues for binding isocitrate (Cupp \& McAlister-Henn, 1993) and lacks two residues for $\mathrm{NADP}^{+}$binding (Cupp \& McAlister-Henn, 1992). The loss of residues for binding $\mathrm{NADP}^{+}$provides nucleotide-binding affinity for AMP, an allosteric activator of IDH (Taylor et al., 2008). In GDH from $T$. thermophilus, amino acid replacements were also found at the nucleotide-binding site of GdhA; however, the GDH activity of GdhA-GdhB(his) was not affected by the nucleotides tested.

As described above, the identity of amino acid sequence is not so high between GdhA and GdhB (46\%). Therefore, it is of interest to elucidate how these two subunits form hetero-oligomers. In homo-hexameric GDH composed of chains A to $\mathrm{F}$ from TmGDH, chain A is in contact with chains B, C and F (Supplementary Fig. S3) by 20, 22 and 24 amino acid residues, respectively. Among the corresponding residues, 15, 17 and 11 residues are conserved between GdhA and GdhB from T. thermophilus (Supplementary Fig. S4). Amino acid conservation at the subunit interface between chains $\mathrm{A}$ and $\mathrm{F}$ is $46 \%(11 / 24)$, which is the same as that of the identity of total protein between GdhA and GdhB (46\%). In contrast, the conservation at the subunit interfaces between chains $\mathrm{A}$ and $\mathrm{B}$ and between chains $\mathrm{A}$ and $\mathrm{C}$ is considerably higher $[75 \%(15 / 20)$ and $76 \%(17 /$ $22)$, respectively]. Based on the difference in conservation of amino acid residues at subunit interfaces, we assume that the twofold symmetry interaction between chains $\mathrm{A}$ and $\mathrm{F}$ is mediated by 'homo'-contact between GdhA and GdhA and between GdhB and GdhB, while the threefold symmetry association between chains $\mathrm{A}$ and $\mathrm{B}$ and chains $\mathrm{A}$ and $\mathrm{C}$ is maintained by not only 'homo'-contact but also GdhA-GdhB 'hetero'-contact. This assumption is consistent with the assembly of GdhA and GdhB into $\mathrm{GdhA}_{4} /$ $\mathrm{GdhB}(\text { his })_{2}$ and $\mathrm{GdhA}_{2} / \mathrm{GdhB}(\text { his })_{4}$ forms.

Ruiz and colleagues have reported that native GDH partially purified from $T$. thermophilus HB8 is only active in oxidative deamination, and not in reductive amination (Ruiz et al., 1998, 2003). Their results and ours are consistent in that GDH exhibits higher oxidative deamination activity than reductive amination activity. We also partially purified GDH from T. thermophilus HB27 and found that it preferred oxidative deamination to reductive amination; however, the enzyme showed distinct reductive amination activity, similar to that of the recombinant GdhA-GdhB(his) complex (data not shown). Between TTC1211 and TTHA1576, three amino acid residues out of 424 residues are different, while five amino acid residues are different between TTC1212 and TTHA1577. The difference in the activity between HB27 and HB8 enzymes might be due to these amino acid substitutions.

Recently, allosteric regulation has been reported for several homo-oligomeric GDHs from bacteria. $P$. aeruginosa GDH is subject to allosteric regulation by arginine and citrate ( $\mathrm{Lu}$ \& Abdelal, 2001). Other examples include S. clavuligerus $\mathrm{GDH}$, which is regulated by aspartate and asparagine (Miñambres et al., 2000), and J. lividum GDH, which is activated by aspartate and arginine (Kawakami et al., 2007). All these GDHs are members of the GDH180s with two functional domains, where the core GDH domain for catalysis is inserted into the regulatory domain. On the other hand, mammalian GDH belonging to the GDH50s is subject to allosteric regulation by leucine (Hudson \& Daniel, 1993), similar to that of T. thermophilus. As for nucleotide effectors, the regulatory mechanism of these compounds has been elucidated by mutational and structural studies (Fang et al., 2002; Smith et al., 2002); however, the mechanism of activation by leucine is unknown, although leucine could bind both to the catalytic site and to a separate regulatory site (Syed \& Engel, 1984). GdhA-GdhB(his) showed negative cooperativity for glutamate in the absence of leucine, while such a feature was not observed in the presence of leucine. This observation suggests that leucine competes with glutamate for binding to the allosteric site, although we cannot discriminate whether the site is for homotropic or heterotropic allostericity. Human GDH is activated by leucine through enhancement of the turnover number (Fang et al., 2002). In $T$. thermophilus GDH, leucine also enhanced the turnover number, in contrast to the case of large GDH from $S$. clavuligerus, where the addition of amino acids decreases the $K_{\mathrm{m}}$ value for substrates while the $k_{\text {cat }}$ value is unaffected. These observations suggest that human GDH and T. thermophilus GDH share similar allosteric mechanisms, although the identity of the amino acid sequence between these enzymes is not high (approximately 34\% between GdhA and human GDHs, and approximately $43 \%$ between GdhB and human GDHs), and there is not a close phylogenetic relationship. At present, the physiological role of the allosteric regulation of $T$. thermophilus GDH by amino acids remains unsolved because various amino acids other than leucine, belonging to a different amino acid family, can activate GDH. This study showed that leucine increases the $k_{\text {cat }}$ value in glutamate synthesis rather than glutamate degradation; however, the leucine-induced increase in the $k_{\text {cat }}$ value of glutamate synthesis is achieved 
with the sacrifice of the $K_{\mathrm{m}}$ value for ammonia, suggesting that GDH still prefers oxidative deamination to reductive amination in vivo. Leucine activates the GDH activity of the GdhA-GdhB(his) hetero-complex more than GdhB alone, suggesting that the amino acid (leucine) is bound at the subunit interface of the hetero-complex. Ongoing crystallographic analysis of GdhA-GdhB and its complex with a hydrophobic amino acid, such as leucine, may clarify not only the regulatory mechanism that acts via hetero-oligomer formation of $T$. thermophilus GDH but also the allosteric regulation of this enzyme.

\section{ACKNOWLEDGEMENTS}

This work was supported in part by grants-in-aid for scientific research from the Ministry of Education, Culture, Sports, Science and Technology Japan and by the Asahi Glass Foundation.

\section{REFERENCES}

Aalén, N., Steen, I. H., Birkeland, N. K. \& Lien, T. (1997). Purification and properties of an extremely thermostable $\mathrm{NADP}^{+}$-specific glutamate dehydrogenase from Archaeoglobus fulgidus. Arch Microbiol 168, 536-539.

Abramoff, M. D., Magelhaes, P. J. \& Ram, S. J. (2004). Image processing with ImageJ. Biophotonics International 11, 36-42.

Baggio, L. \& Morrison, M. (1996). The NAD(P)H-utilizing glutamate dehydrogenase of Bacteroides thetaiotaomicron belongs to enzyme family I, and its activity is affected by trans-acting gene(s) positioned downstream of gdhA. J Bacteriol 178, 7212-7220.

Baker, P. J., Britton, K. L., Rice, D. W., Rob, A. \& Stillman, T. J. (1992), Structural consequences of sequence patterns in the fingerprint region of the nucleotide binding fold. Implications for nucleotide specificity. J Mol Biol 228, 662-671.

Barnes, L. D., Kuehn, G. D. \& Atkinson, D. E. (1971). Yeast diphosphopyridine nucleotide specific isocitrate dehydrogenase. Purification and some properties. Biochemistry 10, 3939-3944.

Bolivar, J. M., Cava, F., Mateo, C., Rocha-Martin, J., Guisan, J. M., Berenguer, J. \& Fernandez-Lafuente, R. (2008). Immobilizationstabilization of a new recombinant glutamate dehydrogenase from Thermus thermophilus. Appl Microbiol Biotechnol 80, 49-58.

Bradford, M. M. (1976). A rapid and sensitive method for the quantitation of microgram quantities of protein utilizing the principle of protein-dye binding. Anal Biochem 72, 248-254.

Camardella, L., Di Fraia, R., Antignani, A., Ciardiello, M. A., di Prisco, G., Coleman, J. K., Buchon, L., Guespin, J. \& Russell, N. J. (2002). The antarctic Psychrobacter sp. TAD1 has two cold-active glutamate dehydrogenases with different cofactor specificities. Characterisation of the $\mathrm{NAD}^{+}$-dependent enzyme. Comp Biochem Physiol A Mol Integr Physiol 131, 559-567.

Cupp, J. R. \& McAlister-Henn, L. (1992). Cloning and characterization of the gene encoding the IDH1 subunit of $\mathrm{NAD}^{+}$-dependent isocitrate dehydrogenase from Saccharomyces cerevisiae. J Biol Chem 267, 16417-16423.

Cupp, J. R. \& McAlister-Henn, L. (1993). Kinetic analysis of $\mathrm{NAD}^{+}$ -isocitrate dehydrogenase with altered isocitrate binding sites: contribution of IDH1 and IDH2 subunits to regulation and catalysis. Biochemistry 32, 9323-9328.

Fang, J., Hsu, B. Y., MacMullen, C. M., Poncz, M., Smith, T. J. \& Stanley, C. A. (2002). Expression, purification and characterization of human glutamate dehydrogenase (GDH) allosteric regulatory mutations. Biochem J 363, 81-87.

Gabriel, J. L., Zervos, P. R. \& Plaut, G. W. (1986). Activity of purified NAD-specific isocitrate dehydrogenase at modulator and substrate concentrations approximating conditions in mitochondria. Metabolism 35, 661-667.

Goldenberg, D. P. (1989). Analysis of protein conformation by gel electrophoresis. In Protein Structure: a Practical Approach, pp. 225250. Edited by T. Creighton. Oxford: IRL Press.

Hamza, M. A. \& Engel, P. C. (2008). Homotropic allosteric control in clostridial glutamate dehydrogenase: different mechanisms for glutamate and $\mathrm{NAD}^{+}$? FEBS Lett 582, 1816-1820.

Henne, A., Bruggemann, H., Raasch, C., Wiezer, A., Hartsch, T., Liesegang, H., Johann, A., Lienard, T., Gohl, O. \& other authors (2004). The genome sequence of the extreme thermophile Thermus thermophilus. Nat Biotechnol 22, 547-553.

Hoseki, J., Okamoto, A., Takada, N., Suenaga, A., Futatsugi, N., Konagaya, A., Taiji, M., Yano, T., Kuramitsu, S. \& Kagamiyama, H. (2003). Increased rigidity of domain structures enhances the stability of a mutant enzyme created by directed evolution. Biochemistry 42, 14469-14475.

Hu, G., Lin, A. P. \& McAlister-Henn, L. (2006). Physiological consequences of loss of allosteric activation of yeast $\mathrm{NAD}^{+}$-specific isocitrate dehydrogenase. J Biol Chem 281, 16935-16942.

Hudson, R. C. \& Daniel, R. M. (1993). L-Glutamate dehydrogenases: distribution, properties and mechanism. Comp Biochem Physiol B 106, 767-792.

Kawakami, R., Sakuraba, H. \& Ohshima, T. (2007). Gene cloning and characterization of the very large NAD-dependent L-glutamate dehydrogenase from the psychrophile Janthinobacterium lividum, isolated from cold soil. J Bacteriol 189, 5626-5633.

Kobayashi, T., Higuchi, S., Kimura, K., Kudo, T. \& Horikoshi, K. (1995). Properties of glutamate dehydrogenase and its involvement in alanine production in a hyperthermophilic archaeon, Thermococcus profundus. J Biochem 118, 587-592.

Koyama, Y., Hoshino, T., Tomizuka, N. \& Furukawa, K. (1986). Genetic transformation of the extreme thermophile Thermus thermophilus and of other Thermus spp. J Bacteriol 166, 338-340.

Laemmli, U. K. (1970). Cleavage of structural proteins during the assembly of the head of bacteriophage T4. Nature 227, 680-685.

Lu, C. D. \& Abdelal, A. T. (2001). The gdhB gene of Pseudomonas aeruginosa encodes an arginine-inducible $\mathrm{NAD}^{+}$-dependent glutamate dehydrogenase which is subject to allosteric regulation. J Bacteriol 183, 490-499.

Miñambres, B., Olivera, E. R., Jensen, R. A. \& Luengo, J. M. (2000). A new class of glutamate dehydrogenases (GDH). Biochemical and genetic characterization of the first member, the AMP-requiring NAD-specific GDH of Streptomyces clavuligerus. J Biol Chem 275, 39529-39542.

Mountain, A., McPherson, M. J., Baron, A. J. \& Wootton, J. C. (1985). The Klebsiella aerogenes glutamate dehydrogenase $(g d h A)$ gene: cloning, high-level expression and hybrid enzyme formation in Escherichia coli. Mol Gen Genet 199, 141-145.

Noor, S. \& Punekar, N. S. (2005). Allosteric NADP-glutamate dehydrogenase from aspergilli: purification, characterization and implications for metabolic regulation at the carbon-nitrogen interface. Microbiology 151, 1409-1419.

Rice, D. W., Baker, P. J., Farrants, G. W. \& Hornby, D. P. (1987). The crystal structure of glutamate dehydrogenase from Clostridium symbiosum at $0.6 \mathrm{~nm}$ resolution. Biochem J 242, 789-795.

Ruiz, J. L., Ferrer, J., Camacho, M. \& Bonete, M. J. (1998). NADspecific glutamate dehydrogenase from Thermus thermophilus HB8: 
purification and enzymatic properties. FEMS Microbiol Lett 159, $15-20$.

Ruiz, J. L., Ferrer, J., Pire, C., Llorca, F. I. \& Bonete, M. J. (2003). Denaturation studies by fluorescence and quenching of thermophilic protein $\mathrm{NAD}^{+}$-glutamate dehydrogenase from Thermus thermophilus HB8. J Protein Chem 22, 295-301.

Sambrook, J., Fritsch, E. F. \& Maniatis, T. (1989). Molecular Cloning: a Laboratory Manual, 2nd edn. Cold Spring Harbor, NY: Cold Spring Harbor Laboratory.

Smith, E. L., Austin, B. M., Blumenthal, K. M. \& Nyc, J. F. (1975). Glutamate dehydrogenase. In The Enzymes, pp. 293-367. Edited by E. D. Boyer. New York: Academic Press.

Smith, T. J., Schmidt, T., Fang, J., Wu, J., Siuzdak, G. \& Stanley, C. A. (2002). The structure of apo human glutamate dehydrogenase details subunit communication and allostery. J Mol Biol 318, 765-777.

Stanley, C. A., Lieu, Y. K., Hsu, B. Y., Burlina, A. B., Greenberg, C. R., Hopwood, N. J., Perlman, K., Rich, B. H., Zammarchi, E. \& Poncz, M. (1998). Hyperinsulinism and hyperammonemia in infants with regulatory mutations of the glutamate dehydrogenase gene. $N$ Engl J Med 338, 1352-1357.

Stillman, T. J., Baker, P. J., Britton, K. L. \& Rice, D. W. (1993). Conformational flexibility in glutamate dehydrogenase. Role of water in substrate recognition and catalysis. J Mol Biol 234, 1131-1139.

Syed, S. E. \& Engel, P. C. (1984). Ox liver glutamate dehydrogenase. The use of chemical modification to study the relationship between catalytic sites for different amino acid substrates and the question of kinetic non-equivalence of the subunits. Biochem J 222, 621-626.

Tanaka, T., Kawano, N. \& Oshima, T. (1981). Cloning of 3 isopropylmalate dehydrogenase gene of an extreme thermophile and partial purification of the gene product. J Biochem 89, 677-682.
Taylor, A. B., Hu, G., Hart, P. J. \& McAlister-Henn, L. (2008). Allosteric motions in structures of yeast $\mathrm{NAD}^{+}$-specific isocitrate dehydrogenase. J Biol Chem 283, 10872-10880.

Thompson, J. D., Higgins, D. G. \& Gibson, T. J. (1994). CLUSTAL W: improving the sensitivity of progressive multiple sequence alignment through sequence weighting, position-specific gap penalties and weight matrix choice. Nucleic Acids Res 22, 4673-4680.

Turano, F. J., Thakkar, S. S., Fang, T. \& Weisemann, J. M. (1997). Characterization and expression of $\mathrm{NAD}(\mathrm{H})$-dependent glutamate dehydrogenase genes in Arabidopsis. Plant Physiol 113, 1329-1341.

Veronese, F. M., Nyc, J. F., Degani, Y., Brown, D. M. \& Smith, E. L. (1974). Nicotinamide adenine dinucleotide-specific glutamate dehydrogenase of Neurospora. I. Purification and molecular properties. J Biol Chem 249, 7922-7928.

Watson, D. H. \& Wootton, J. C. (1978). Subunit ratios of separated hybrid hexamers of Neurospora NADP-specific glutamate dehydrogenase containing complementing mutationally modified monomers. Biochem J 175, 1125-1133.

Wen, Z. \& Morrison, M. (1996). The NAD(P)H-dependent glutamate dehydrogenase activities of Prevotella ruminicola B14 can be attributed to one enzyme (GdhA), and $g d h A$ expression is regulated in response to the nitrogen source available for growth. Appl Environ Microbiol 62, 3826-3833.

West, D. J., Tuveson, R. W., Barratt, R. W. \& Fincham, J. R. (1967). Allosteric effects in nicotinamide adenine dinucleotide phosphatespecific glutamate dehydrogenase from Neurospora. J Biol Chem 242, 2134-2138.

Edited by: D. J. Kelly 